\title{
Polarimetry of GPS and CSS Sources
}

\author{
W. D. Cotton ${ }^{1}$, D. Dallacasa ${ }^{2,3}$, C. Fanti ${ }^{2,3}$, R. Fanti ${ }^{2,3}$, A. R. Foley ${ }^{4}$, \\ R. T. Schilizzi ${ }^{5,6}$, R. Spencer ${ }^{7}$, D. J. Saikia ${ }^{8}$ and S. Garrington ${ }^{7}$ \\ ${ }^{1}$ National Radio Astronomy Observatory, 520 Edgemont Road, \\ Charlottesville, VA 22903-2475 USA \\ bcotton@nrao.edu \\ ${ }^{2}$ Istituto di Radioastronomia del CNR, Via P. Gobetti 101, I-40129, Bologna, Italy \\ ddallaca@ira.cnr.it \\ ${ }^{3}$ Dipartimento di Astronomia, Universitá di Bologna, via Ranzani 1, I-40127 Bologna, Italy \\ cfanti@astbo1.cnr.it \\ rfanti@astbo1.bo.cnr.it \\ ${ }^{4}$ Netherlands Foundation for Research in Astronomy, Postbus 2, \\ NL-7990 AA Dwingeloo, The Netherlands \\ foley@ astron.nl \\ ${ }^{5}$ Joint Institute for VLBI in Europe, Postbus 2, NL-7990 AA Dwingeloo \\ ${ }^{6}$ Sterrenwacht Leiden, Postbus 9513, NL-2300 RA Leiden, The Netherlands \\ schilizzi@jive.nl \\ ${ }^{7}$ Nuffield Radio Astronomy Laboratories, Jodrell Bank, Cheshire SK11 9D1, UK \\ res@jb.man.ac.uk \\ stg@jb.man.ac.uk \\ 8 Tata Institute of Fundamental Reasearch, National Centre for Radio Astrophysics, \\ Pune 411 007, India \\ djs@ncra.tifr.res.in
}

Received 2002 July 1, accepted 2002 September 16

\begin{abstract}
The role of radio polarimetry in the understanding of GPS and CSS sources is explored. After an initial discussion of what can be learned from polarimetry, the expectations of a simple evolutionary sequence of GPS/CSO to CSS to FR I/FR II sources are explored. Observational results are then compared with the expectations. Conclusions include: the GPS category is likely not a single homogeneous class of objects; Faraday depth effects are very strong inside the inner $3 \mathrm{kpc}$ of CSS and CSO sources; in at least 3C 138 the Faraday screen has very fine scale (subparsec) structure; and there is evidence for increased ionisation near bends in some CSS jets probably due to jet-ISM interaction. New results on 3C 138, 3C 43, and $3 \mathrm{C} 454$ are given.
\end{abstract}

Keywords: polarisation — galaxies: active — galaxies: ISM — galaxies: jets — radio continuum: galaxies — radio continuum: ISM

\section{Introduction}

Measurements of the polarisation state of the radio emission from gigahertz peaked spectrum (GPS) and compact steep spectrum (CSS) sources can give a great deal of information about the physical conditions inside and in front of the emitting region.

At wavelengths where the source is optically thin to synchrotron self absorption, the emitted radiation is strongly linearly polarised and the $E$-vector of the emission is perpendicular to the direction of the magnetic field. In regions where the field is well ordered, the fractional linear polarisation can approach $70 \%$. Disordered magnetic fields will reduce the fractional polarisation.

An effect that is even more important for GPS and CSS sources which reside inside their host galaxies is Faraday rotation. A birefringent medium such as a magnetised thermal plasma has a different index of refraction for the two circularly polarised modes which has the effect of rotating the orientation of the linearly polarised component of the transmitted radiation. The amount of rotation is given by (Pacholczyk 1970)

$$
\Delta \Phi=\frac{0.93 \times 10^{6}}{(2 \pi \nu)^{2}} \int N_{\mathrm{e}} H_{\|} d s
$$

where $v$ is the radio frequency of the emission, $N_{\mathrm{e}}$ is the electron density, and $H_{\|}$is the component of the magnetic field parallel to the direction of propagation. The 'rotation measure' is the amount of Faraday rotation expressed in $\operatorname{rad~} \mathrm{m}^{-2}$.

As can be seen from this equation, the Faraday rotation is proportional to the wavelength squared and can be determined from observations at a number of wavelengths, giving an integral along the line of sight of the thermal electron density times the parallel component of the magnetic field. Strong variations of Faraday rotation across the telescope beam will reduce or 'depolarise' the observed fractional polarisation. This effect can, and frequently does, completely depolarise regions of emission, especially at longer wavelengths. 


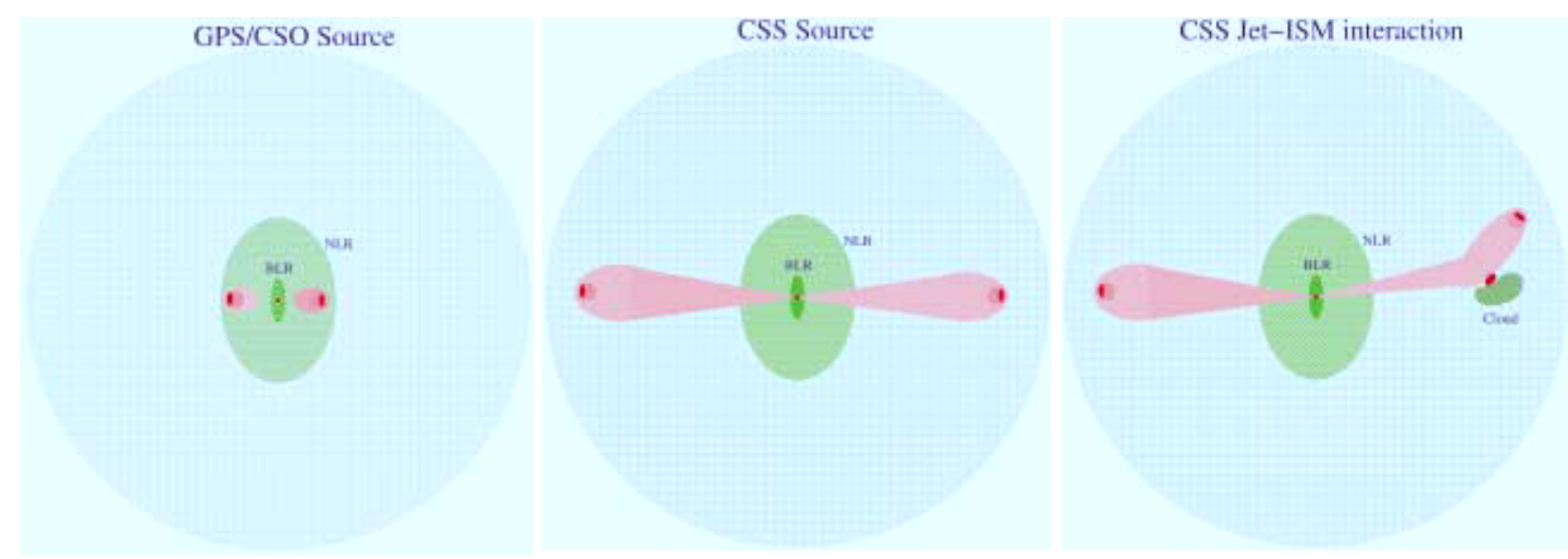

Figure 1 Cartoon representations of the relationship between GPS and CSS sources and the host galaxy. Left: GPS/CSO source entirely interior to NLR. Centre: CSS source larger than NLR. Right: CSS jet bent by interaction with a cloud in the host ISM.

\section{Expectations}

The following discussion is in the context of the commonly accepted scenario (O'Dea 1998) that GPS/CSO galaxies are recently born radio galaxies that will grow into CSS sources that eventually grow into the classical extended radio sources. According to this scenario, the GPS/CSO and CSS sources reside inside relatively gasrich host galaxies. If the ISM in the host is sufficiently dense, it may block the radio source from expanding into the intergalactic medium. In any case, interaction between the radio jet and the ISM should be evident as optical line emission and Faraday effects in the ISM. These cases are illustrated in Figure 1. HST observations of a number of CSS sources (Axon et al. 2000) show optical line emission associated with the radio jets confirming the jet-ISM interaction.

Since GPS/CSO sources are thought to be very young, they will reside in the inner regions of the host and will largely be confined to the narrow line region (NLR) with its relatively large column densities of plasma. Very strong Faraday effects are to be expected in these sources, likely completely depolarising them at longer wavelengths. Also, the cores in CSO sources are generally very weak so the expectation is that any variations should be slow and weak.

CSS sources, while still subgalactic in size, frequently exceed the size of the NLR, so Faraday effects in the outer parts of these sources should be less than in the inner regions. However, jet cloud interactions anywhere in the galaxy may give rise to local enhancements in the plasma density, hence local Faraday rotation. As jet-cloud interactions will likely deflect the jet, enhanced Faraday effects (either rotation or depolarisation) are expected near the bends that are common in CSS jets. This is illustrated in the rightmost panel of Figure 1.

\section{Observations: GPS Sources}

One measure of the total Faraday effects on a source is the integrated polarisation of the radio emission from the source at a low frequency. In order to consider this issue, the GPS sample of Stanghellini et al. (1998) was matched

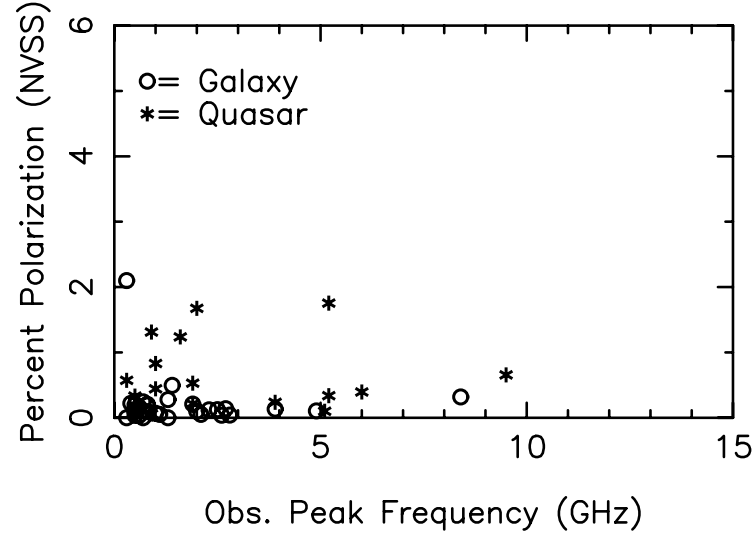

Figure 2 Fractional polarisation at $1.4 \mathrm{GHz}$ from the NVSS survey plotted against the frequency of the peak in the spectrum for the GPS sample of sources from Stanghellini et al. (1998).

with the NVSS catalogue (Condon et al. 1998) which contains integrated total and linearly polarised intensities. The expectation is that the smaller sources will have lower fractional polarisation due to the increased Faraday depth of the source. The sources in this sample do not all have well measured sizes but O'Dea (1998) has shown an inverse correlation between the frequency of the peak in the spectrum and the overall size of the source. The results of the comparison of fractional polarisation against the frequency of the peak in the spectrum are shown in Figure 2 where galaxies are shown as open circles and quasars as asterisks. With the exception of one galaxy with a low peak frequency (hardly a GPS source) all the galaxies have very low polarisation while many of the quasars have some polarisation regardless of the peak frequency.

If the GPS sources really are very young lobedominated radio galaxies similar to the CSOs, then they should not have short term variations in total intensity or polarisation. The University of Michigan Radio Astronomy Observatory (UMRAO) has been monitoring a number of GPS sources for several decades. ${ }^{1}$

\footnotetext{
${ }^{1}$ http://www.astro.lsa.umich.edu/obs/radiotel/umrao.html
} 
Several of the GPS objects being monitored by UMRAO show rather more variability than is expected in the young radio galaxy scenario (M. Aller 2002, private communication). 4C 66.22 shows only weak fluctuations in total intensity but large time variations in polarised intensity and orientation. The source B1127-145 is variable in total and polarised intensity and orientation but mostly maintains its GPS spectrum; however, for much of 1999 its spectrum was flat between 4.9 and $14.5 \mathrm{GHz}$.

Circular polarisation in any extragalactic radio source is very weak but the most strongly circularly polarised source in the study by Homan, Attridge, \& Wardle (2001) - B0743-006 — is also in the sample of Stanghellini et al. (1998). The observed circular polarisation is possibly the result of Faraday conversion of linear into circular polarisation by thermal plasma mixed with the synchrotron emitting relativistic plasma.

A VLBI study of $21 \mathrm{CSO}$ sources at $8.4 \mathrm{GHz}$ by Peck \& Taylor (2000) did not detect linear polarisation in any and set upper limits of about $0.5 \%$. This suggests that the low polarisation is not due to beam depolarisation and the relatively high frequency suggests a very high Faraday depth if the low polarisation is the result of Faraday depolarisation.

\section{Observations: CSS Sources}

A sample of CSS and GPS sources is given in Fanti et al. (2001). Since CSS sources are generally comparable or larger than the size of the NLR, the Faraday depth should be less, allowing larger amounts of linear polarisation to escape. A plot of the NVSS fractional polarisation against largest linear size of this sample is shown in Figure 3. This figure has a rather unexpected result: sources smaller than $6 \mathrm{kpc}$ in extent are very weakly polarised, whereas sources larger than this can have up to several per cent integrated linear polarisation. Since the linear size includes both the jet and counter-jet sides, this suggests that there is an abrupt change in the properties of the ISM at a radius of approximately $3 \mathrm{kpc}$.

It has long been recognised that CSS sources were prominent among those with very large integrated rotation measures (Taylor, Inoue, \& Tabara 1992; Inoue et al. 1995; Junor et al. 1996). High rotation measures have been found in the nuclear regions of some CSS sources, for example OQ 172 (Taylor, Venturi, \& Udomprasert 1998) and 3C 138 (Cotton et al. 1997). Furthermore, the high rotation measure component in $3 \mathrm{C} 138$ has a detectable motion and the variable rotation measure indicates very fine structure in the Faraday screen. Preliminary results of a monitoring project of the Faraday rotation of this component are shown in Figure 4. These variations of Faraday rotation suggest that there is structure in the Faraday screen on the subparsec scale.

Regions of high rotation measure have also been reported near the bends or other jet-ISM interactions in a number of CSS jets, for example 3C 119 (Flatters 1998; Nan et al. 1999), 3C 216 (Lüdke et al. 1998; Taylor et al. 1998), and B0548+165 (Mantovani et al. 2002).

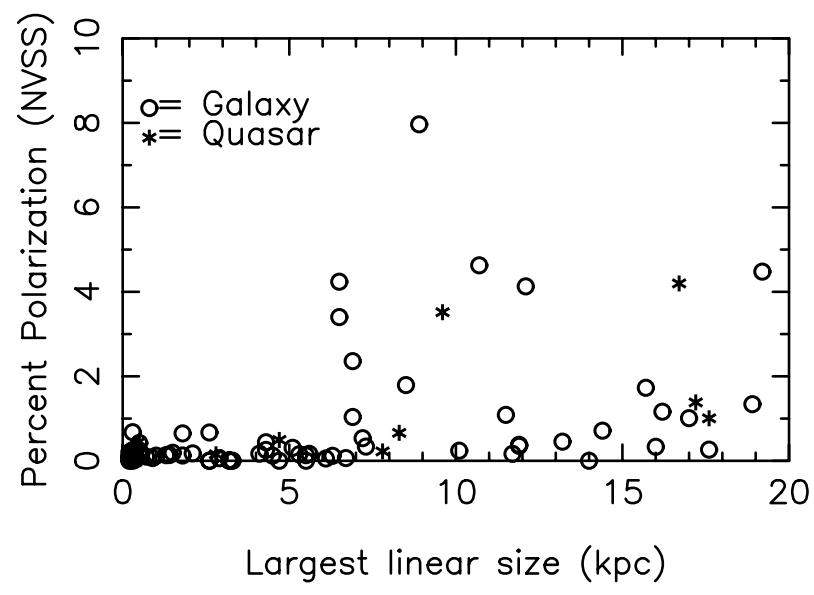

Figure 3 Fractional polarisation at $1.4 \mathrm{GHz}$ from the NVSS survey plotted against the largest angular size for the sample of CSS/GPS sources from Fanti et al. (2001).
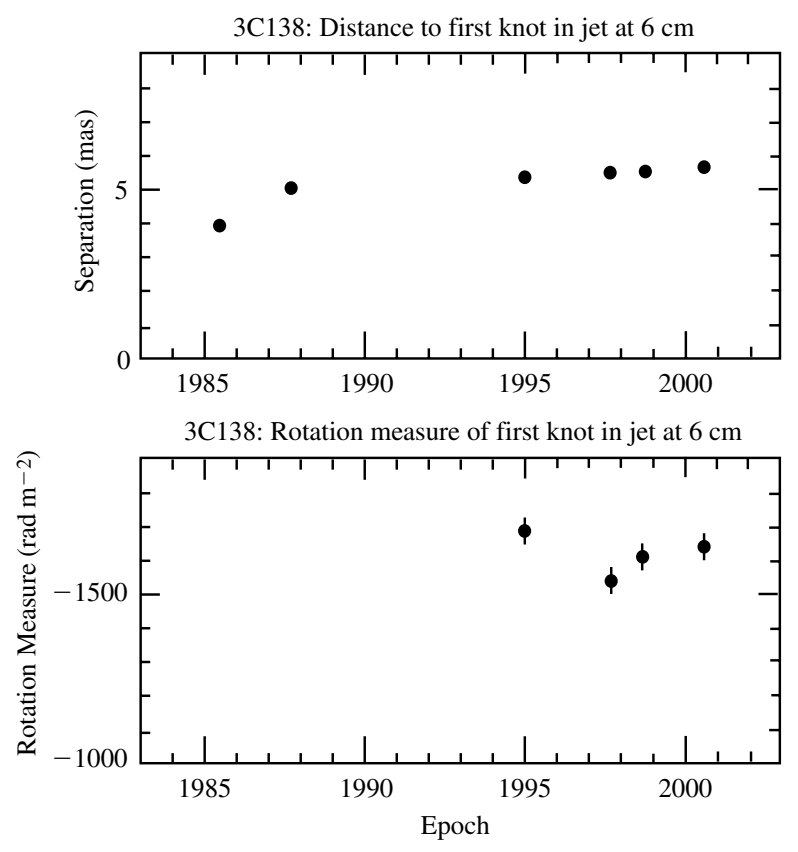

Figure 4 Separation of the polarised nuclear feature from the core (upper) and its Faraday rotation (lower) in the observer's frame of a polarised, moving component near the nucleus of the CSS quasar $3 \mathrm{C} 138$. In the source frame the rotation measures are a factor $\left((1+z)^{2}\right)$ of 3.1 higher.

Rotation measure observations of two CSS quasars with bent jets by the VLBA at $18 \mathrm{~cm}$ wavelength are shown in Figure 5. 3C 43 (Figure 5, left) is weakly polarised but shows enhanced Faraday rotation in the region of the bend in the jet. The other source, 3C 454 (Figure 5, right), is more strongly polarised but has relatively large rotation measures from the core (top) until the jet reaches the southern knot where the jet is deflected to the west. At this knot, the Faraday rotation is lower than further upstream in the jet. This could possibly be caused by the jet nearly drilling through the deflecting cloud reducing the Faraday screen. 

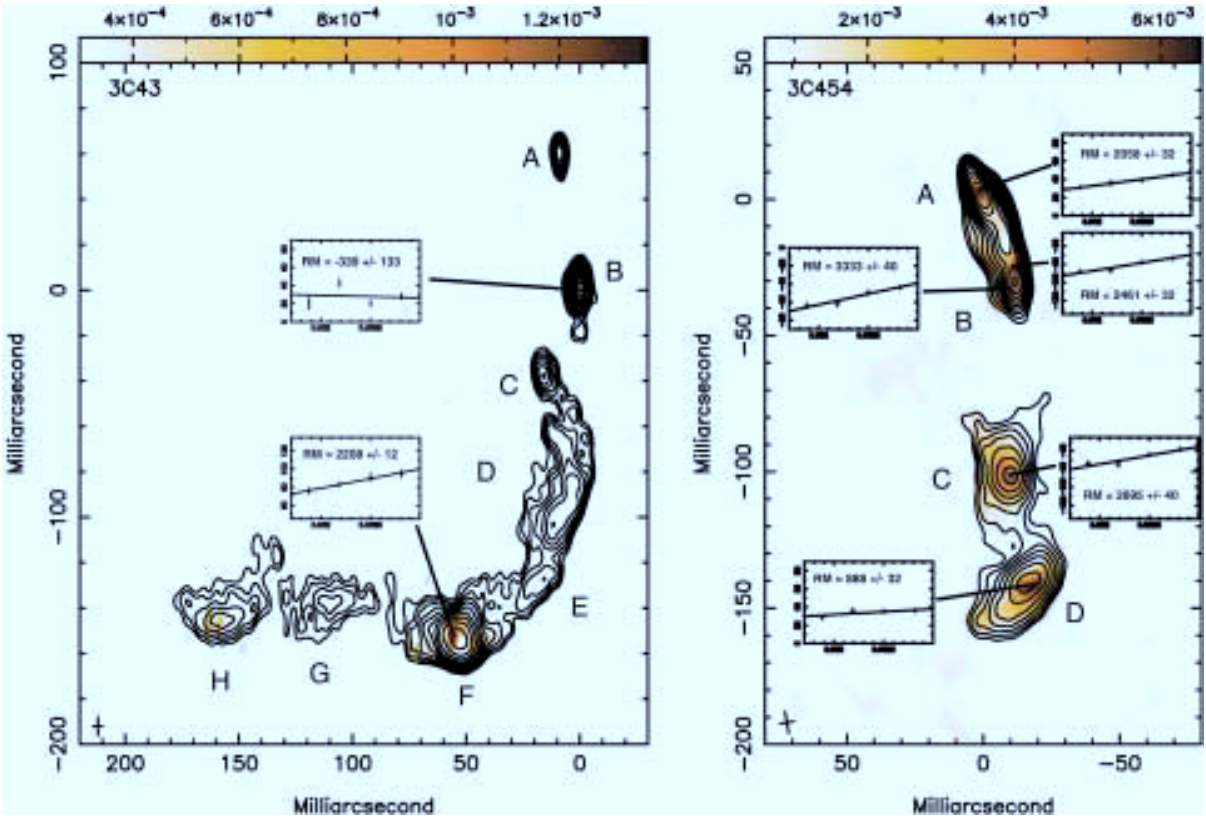

Figure 5 Left: Total intensity contours of $3 \mathrm{C} 43$ at $1.7 \mathrm{GHz}$ with polarised intensity in colour. Inset plots show the run of polarisation angle with wavelength squared at selected locations. The cross in the lower left indicates the resolution. Right: As for left but for 3C 454

A similar effect has been seen in B1524-136 (Mantovani et al. 2002).

\section{Conclusions}

The polarisation properties of the GPS/CSO sources are in good agreement with the expectations of the young radio galaxy scenario: they are strongly depolarised. However, there are a number of sources, especially quasars, with GPS spectra which show significant linearly polarised emission which may also be time variable. It is not clear that these sources fit into the standard evolutionary picture as newly born radio galaxies and may simply be blazars with a single dominant component or intervening free-free absorbing screen.

The well-studied CSS sources have polarisation properties in agreement with the general expectations of the evolutionary scenario; smaller sources are more depolarised than larger ones. However, this distinction does not appear to be a gradual increase in polarisation with source size but a discontinuity at a total source size of about $6 \mathrm{kpc}$. This needs further study but could be related to the outer boundary of the NLR. Observations of 3C 138 suggest very fine scale structure in the Faraday screen.

A number of CSS sources with bent jets appear to have increased Faraday rotation associated with the bends in the jets, supporting the presumption that much of the plasma in the objects is due to jet-ISM interactions. New data are presented on 3C 43 and $3 \mathrm{C} 454$.

\section{Acknowledgments}

The National Radio Astronomy Observatory is a facility of the National Science Foundation operated under cooperative agreement by Associated Universities, Inc.

\section{References}

Axon, D. J., Capetti, A., Fanti, R., Morganti, R., Robinson, A., \& Spencer, R. 2000, AJ, 120, 2284

Condon, J. J., Cotton, W. D., Greisen, E. W., Yin, Q. F., Perley, R. A., Taylor, G. B., \& Broderick, J. J. 1998, AJ, 115, 1693

Cotton, W. D., Dallacasa, D., Fanti, C., Fanti, R., Foley, A. R., Schilizzi, R. T., \& Spencer, R. E. 1997, A\&A, 325, 493

Fanti, C., Pozzi, F., Dallacasa, D., Fanti, R., Gregorini, L., Stanghellini C., \& Vigotti, M. 2001, A\&A, 3369, 380

Flatters, C. 1998, in Radio Emission from Galactic and Extragalactic Compact Sources, IAU Colloquium 164, ASP Conference Series, ed. J. A. Zensus, G. B. Taylor, \& J. M. Wrobel (San Francisco: ASP), 109

Homan, D. C., Attridge, J. M., \& Wardle, J. F. C. 2001, ApJ, 556, 113

Inoue, M., Tabara, H., Kato, T., \& Aizu, K. 1995, PASJ, 4, 725

Junor, W., Mantovani, F., Peck, A., Saikia, D., \& Salter, C. 1996, in Extragalactic Radio Sources, IAU Colloquium 175 ed. L. Padrelli, R. D. Ekers, \& C. Fanti (Kluwer Academic Publishers), 79

Lüdke, E., Garrington, S. T., Spencer, R. E., Akujor, C. E., Muxlow, T. W. B., Sanghera, H. S., \& Fanti, C. 1998, MNRAS, 299, 467

Mantovani, F., Junor, W., Ricci, R., Saikia, D. J., Salter, C., \& Bondi, M. 2002, A\&A, 389, 58

Nan, R., Gabuzda, D. C., Kameno, S., Schilizzi, R. T., \& Inoue, M. 1999, A\&A, 344, 402

O’Dea, C. P. 1998, PASP, 110, 493

Pacholczyk, A. G. 1970, Radio Astrophysics (San Francisco: Freeman)

Peck, A. B., \& Taylor, G. 2000, in Proceedings of the 5th EVN Symposium, ed. A. Polatidis, J. Conway, \& R. Booth (Onsala Space Observatory), 95

Stanghellini, C., O'Dea, C. P., Dallacasa, D., Baum, S. A., Fanti, R., \& Fanti, C. 1998, A\&AS, 131, 303

Taylor, G., Inoue, I., \& Tabara, H. 1992, A\&A, 264, 421

Taylor, G., Venturi, T., \& Udomprasert, P. S. 1998, in Radio Emission from Galactic and Extragalactic Compact Sources, IAU Colloquium 164, ASP Conference Series 144, ed. J. A. Zensus, G. B. Taylor, \& J. M. Wrobel (San Francisco: ASP), 1113 